\title{
Light-gravitino production at hadron colliders
}

\author{
Jaewan Kim, ${ }^{1,}{ }^{*}$ Jorge L. Lopez, ${ }^{2, \dagger}$ D. V. Nanopoulos, ${ }^{1,3,}$ Raghavan Rangarajan, ${ }^{1, \S}$ and A. Zichichi ${ }^{4}$ \\ ${ }^{1}$ Astroparticle Physics Group, Houston Advanced Research Center (HARC), The Mitchell Campus, The Woodlands, Texas 77381 \\ ${ }^{2}$ Bonner Nuclear Lab, Department of Physics, Rice University, 6100 Main Street, Houston, Texas 77005 \\ ${ }^{3}$ Center for Theoretical Physics, Department of Physics, Texas A\&M University, College Station, Texas $77843-4242$ \\ and Academy of Athens, Chair of Theoretical Physics, Division of Natural Sciences 28 Panepistimiou Avenue, 10679 Athens, Greece \\ ${ }^{4}$ University and INFN, Bologna, Italy \\ and CERN, 1211 Geneva 23, Switzerland
}

(Received 14 July 1997; published 4 December 1997)

\begin{abstract}
We consider the production of gravitinos $(\widetilde{G})$ in association with gluinos $(\widetilde{g})$ or squarks $(\widetilde{q})$ at hadron colliders, including the three main subprocesses: $q \bar{q} \rightarrow \widetilde{g} \widetilde{G}$, and $q g \rightarrow \widetilde{q} \widetilde{G}$, and $g g \rightarrow \widetilde{g} \widetilde{G}$. These channels become enhanced to the point of being observable for sufficiently light gravitino masses $\left(m_{\tilde{G}}<10^{-4} \mathrm{eV}\right)$, as motivated by some supersymmetric explanations of the Collider Detector at Fermilab ee $\gamma \gamma+E_{T \text {,miss }}$ event. The characteristic signal of such events would be monojets, as opposed to dijets obtained in the more traditional supersymmetric process $p \bar{p} \rightarrow \widetilde{g} \widetilde{g}$. Searches for such events at the Fermilab Tevatron can impose lower limits on the gravitino mass. In the appendixes, we provide a complete set of Feynman rules for the gravitino interactions used in our calculation. [S0556-2821(98)02101-8]
\end{abstract}

PACS number(s): 12.60.Jv, 04.65.+e, 14.80.Ly

\section{INTRODUCTION}

Supersymmetric models where the gravitino $(\widetilde{G})$ is the lightest supersymmetric particle have been considered in the literature for some time [1-3]. More recently they have enjoyed a resurgence motivated by new models of dynamical supersymmetry breaking [4] and by possible explanations $[5,6]$ of the puzzling Collider Detector at Fermilab (CDF) $e e \gamma \gamma+E_{T, \text { miss }}$ event [7]. Most of the recent effort has been devoted to studying the new signals for supersymmetry via electroweak-interaction processes, that accompany such scenarios by virtue of the newly allowed $\chi \rightarrow \gamma \widetilde{G}$ decay (i.e., diphoton signals from $e^{+} e^{-} \rightarrow \chi \chi$ and analogous signals in $p \bar{p}$ production of a variety of supersymmetric channels) [4$6]$, or via direct gravitino production (i.e., single photon signals from $e^{+} e^{-} \rightarrow \chi \widetilde{G}[8,9]$ or $\left.p \bar{p} \rightarrow \chi \widetilde{G}, \chi^{ \pm} \widetilde{G}[9]\right)$. Much less emphasis has been placed on strong-interaction processes at hadron colliders [3,10].

An important part of this phenomenological effort should be directed at obtaining direct experimental information on the gravitino mass. However, this information will not come from the kinematical effects of such a very light particle, but instead from the dynamical effect that each interaction vertex involving gravitinos is inversely proportional to the gravitino mass. Lower bounds on the gravitino mass from singlephoton searches at LEP already exist, but are limited by kinematics at the CERN $e^{+} e^{-}$collider LEP $1[8,9]$ and by dynamics at LEP 2 [9]. Limits on the gravitino mass based

\footnotetext{
*Electronic address: jaewan@diana.tdl.harc.edu

†Present address: Shell E\&P Technology Company, Bellaire Technology Center, P. O. Box 481, Houston, TX 77001-0481. Electronic address: jorge@shellus.com

*Electronic address: dimitri@phys.tamu.edu

${ }^{\S}$ Electronic address: raghu@diana.tdl.harc.edu
}

on monojet and multijet final states at the Tevatron are obtained in Ref. [10]. Limits also exist from astrophysical and cosmological considerations [11]. These limits are further revised in Ref. [12,13]. More stringent constraints from the $Z$ decay width have also been obtained in Ref. [13].

The purpose of this paper is to examine the manifestation of light gravitinos in the context of hadron colliders. ${ }^{1}$ Two gravitino-mass-dependent processes involving the strong interactions come to mind:

$$
\begin{gathered}
p \bar{p} \rightarrow \widetilde{g g}, \\
p \bar{p} \rightarrow \widetilde{g} \widetilde{G}, \widetilde{q} \widetilde{G} .
\end{gathered}
$$

The first process $(p \bar{p} \rightarrow \widetilde{g} \widetilde{g}$ )proceeds via the usual supersymmetric QCD diagrams, but receives in addition contributions from new gravitation-induced diagrams involving exchange of gravitinos in the $t$ and $u$ channels [10]. For sufficiently light gravitino masses $\left(m_{\tilde{G}} \lesssim 10^{-3} \mathrm{eV}\right)$, the gravitationinduced contributions dominate the supersymmetric QCD ones, otherwise the reverse is true. This process is also kinematically constrained at the Tevatron to $m_{\tilde{g}} \lesssim 250 \mathrm{GeV}$. The second process $(p \bar{p} \rightarrow \widetilde{g} \widetilde{G}, \widetilde{q} \widetilde{G})$ only receives contributions from gravitation-induced diagrams and is therefore only relevant for sufficiently light gravitino masses. However, its kinematical reach at the Tevatron is much greater $\left(m_{\tilde{g}}, m_{\tilde{q}}\right.$ $\$ 500 \mathrm{GeV}$ ). In Ref. [10], only the process $p \bar{p} \rightarrow \widetilde{g} G$ via gluon fusion was studied. Here we consider the $\widetilde{q} \widetilde{G}$ final state as well, and also include the $q \bar{q}$ and $q g$ channels. We find that for sufficiently light gravitinos $\left(m_{\widetilde{G}} \lesssim 3\right.$

\footnotetext{
${ }^{1}$ For definiteness, in what follows we will concentrate on the Fermilab Tevatron $(p \bar{p})$ collider, although the discussion applies with little modification to the case of the CERN Large Hadron Collider (LHC) $(p p)$ too.
} 

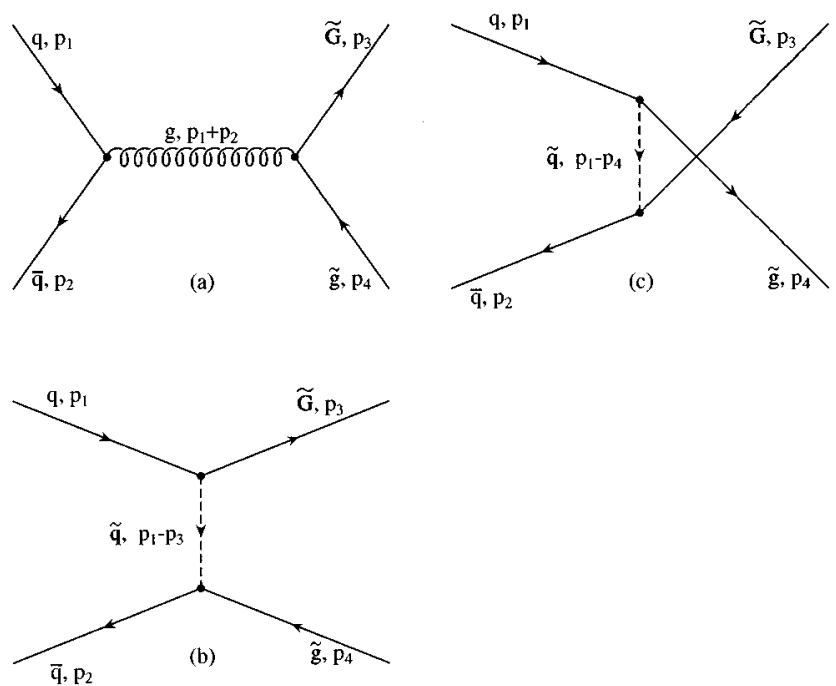

FIG. 1. Feynman diagrams for subprocess $q \bar{q} \rightarrow \widetilde{g} \widetilde{G} . p_{1}$ and $p_{2}$ are incoming momenta and $p_{3}$ and $p_{4}$ are outgoing momenta. Arrows on scalars indicate direction of momentum and flow of particle flavor.

$\left.\times 10^{-5}\right) \mathrm{eV}$, the second process has a larger cross section even within the kinematical reach of the first process $\left(m_{\tilde{g}}\right.$ $\$ 250 \mathrm{GeV}$ ). Some of our results disagree with those of Ref. [10].

It is also important to consider the gluino decay modes. For sufficiently light gravitinos $\left(m_{\tilde{G}} \lesssim 10^{-3} \mathrm{eV}[14]\right)$ these are dominated by $\widetilde{g} \rightarrow g \widetilde{G}$; otherwise they proceed in complicated ways of which $\widetilde{g} \rightarrow q \bar{q} \chi$ is an example. We then obtain a partitioning of the $\left(m_{\widetilde{G}}, m_{\tilde{g}}\right)$ space into four regions of "low or high" values of the parameters, roughly delimited by $m_{\widetilde{G}} \sim 10^{-3} \mathrm{eV}$ and $m_{\widetilde{g}} \sim 250 \mathrm{GeV}$. Note that in each of these regions we expect a signal dominated by a different number of jets. These facts are summarized in the following sketch:

$$
\begin{gathered}
\text { high } m_{\widetilde{G}}\left(\begin{array}{c}
p \bar{p} \rightarrow \widetilde{g g} \\
\tilde{g} \rightarrow q \bar{q} \chi \\
4 \text { jets }
\end{array}\right)\left(\begin{array}{c}
p \bar{p} \rightarrow \widetilde{g G} \\
\widetilde{g} \rightarrow q \bar{q} \chi \\
2 \text { jets }
\end{array}\right), \\
\text { low } m_{\widetilde{G}}\left(\begin{array}{c}
p \bar{p} \rightarrow \widetilde{g} \widetilde{g} \\
\tilde{g} \rightarrow g \widetilde{G} \\
2 \text { jets }
\end{array}\right)\left(\begin{array}{c}
p \bar{p} \rightarrow \widetilde{g} \widetilde{G} \\
\tilde{g} \rightarrow g \widetilde{G} \\
1 \text { jet }
\end{array}\right), \\
\text { low } m_{\tilde{g}}, \text { high } m_{\tilde{g}} .
\end{gathered}
$$

The sparse literature on this subject contains explicit expressions only for the $g g$ initiated subprocesses $(g g \rightarrow \widetilde{g} \widetilde{g}, \widetilde{g})$ $[10]$ and phenomenological analyses $[3,10]$ only for the left half (i.e., "low $m_{\widetilde{g}}$ ") of the above table.

The above comments and processes generally apply to squarks as well, with gluinos replaced by squarks $(\widetilde{g} \rightarrow \widetilde{q})$ and gluons replaced by quarks $(g \rightarrow q)$. A sample squark decay for heavier gravitinos is $\widetilde{q} \rightarrow q \chi$.

In Sec. II we present the cross sections for different channels at a hadron collider giving a monojet signal and an outgoing gravitino. In Sec. III we present a discussion of our
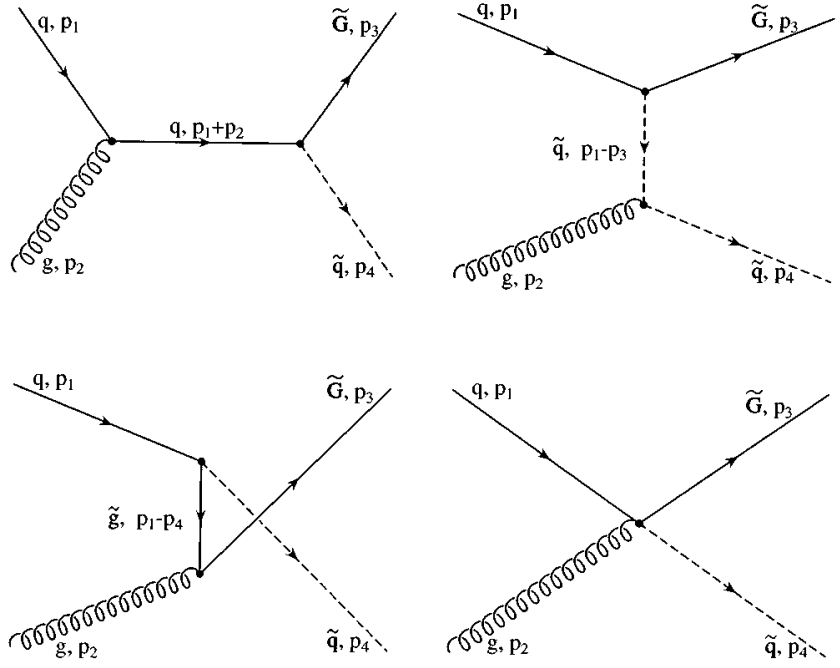

FIG. 2. Feynman diagrams for subprocess $q g \rightarrow \widetilde{q} \widetilde{G} \cdot p_{1}$ and $p_{2}$ are incoming momenta and $p_{3}$ and $p_{4}$ are outgoing momenta. Arrows on scalars indicate direction of momentum and flow of particle flavor.

numerical results. In the appendixes we provide a derivation of the interaction Lagrangian involving gravitinos, stating clearly the conventions we use. We include the Feynman rules relevant to our calculation and also provide comparisons with others using different conventions.

In what follows we have not included processes involving the scalar partners of the goldstino $(S$ and $P)$. Such processes can also give rise to monojet signals in a hadron collider, if the $S$ and $P$ are light, and shall be addressed in future work. Limits on the gravitino mass that may be obtained using our results will thus be conservative.

Processes involving gravitinos suffer unitarity violation at high energies due to the nonrenormalizability of the supergravity Lagrangian [15]. To preserve unitarity until energies
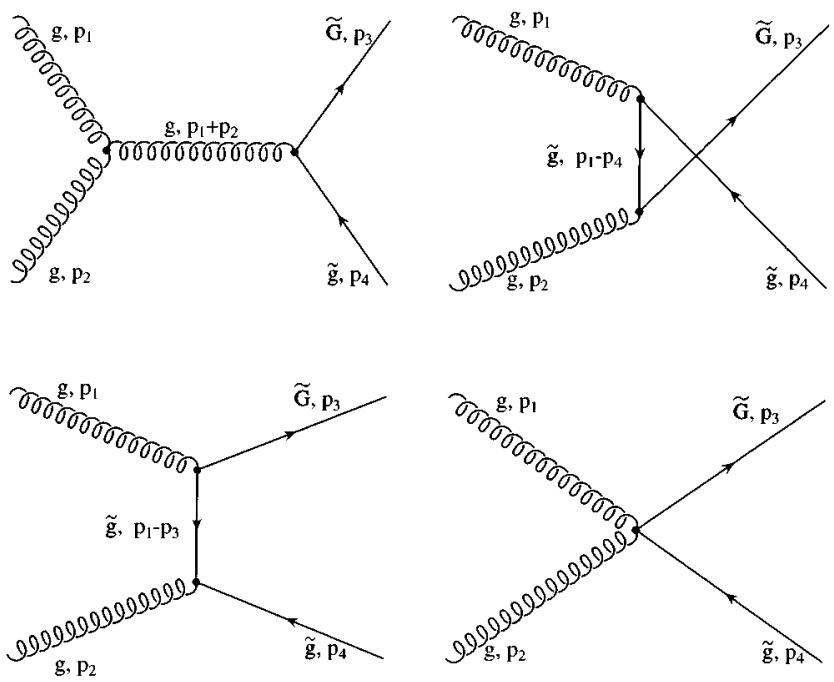

FIG. 3. Feynman diagrams for subprocess $g g \rightarrow \widetilde{g} \widetilde{G} \cdot p_{1}$ and $p_{2}$ are incoming momenta and $p_{3}$ and $p_{4}$ are outgoing momenta. Arrows on scalars indicate direction of momentum and flow of particle flavor. 
of the order of a few TeV typically requires $m_{\tilde{G}} \gtrsim 10^{-6} \mathrm{eV}$. Hence, we shall take $10^{-6} \mathrm{eV}$ as our lower bound on the gravitino mass in what follows.

\section{ANALYTICAL RESULTS}

In $p \bar{p}$ collision there are three parton sub-processes giving rise to a single jet along with a gravitino. As we mention above, the jet is initiated by either an outgoing gluino or squark and the undetected gravitino is associated with missing transverse energy. The different parton subprocesses are $q \bar{q} \rightarrow \widetilde{g} \widetilde{G}, q g \rightarrow \widetilde{q} \widetilde{G}$, and $g g \rightarrow \widetilde{g} \widetilde{G}$. Below we present the differential cross section $d \hat{\sigma} / d t$ for each subprocess. The Feynman diagrams for each sub-process are provided in Figs. 1, 2, and 3, respectively. The hadronic cross section $\sigma(S)$ is obtained by convoluting the subprocess cross section $\hat{\sigma}_{i, j}$ of partons $i, j$ with parton distribution functions $f_{i}(x, Q)$. (Because of the relation between Mandelstam variables $s, t$ and $u$, the partonic cross sections below may be expressed differently.)

The differential cross section for the subprocess $q \bar{q} \rightarrow \widetilde{g} \widetilde{G}$ is given by

$$
\begin{aligned}
d \hat{\sigma} / d t= & \frac{1}{4 \times 9 \times 16 \pi s^{2}} \frac{4 g_{s}^{2}}{M^{2} m_{3 / 2}^{2}}\left[\frac{4 s\left(s-m_{\tilde{g}}^{2}\right)\left(t^{2}+u^{2}\right)}{3 s^{2}}\right. \\
& +\sum_{j=1,2} \frac{2 t^{3}\left(t-m_{\widetilde{g}}^{2}\right)}{3\left[t-m_{\widetilde{q}_{j}}^{2}\right]^{2}}+\sum_{j=1,2} \frac{2 u^{3}\left(u-m_{\widetilde{g}}^{2}\right)}{3\left[u-m_{\widetilde{q}_{j}}^{2}\right]^{2}} \\
& \left.-\sum_{j=1,2} \frac{4 s t^{3}}{3 s\left[t-m_{\widetilde{q}_{j}}^{2}\right]}-\sum_{j=1,2} \frac{4 s u^{3}}{3 s\left[u-m_{\widetilde{q}_{j}}^{2}\right]}\right] .
\end{aligned}
$$

Above $M=\left(8 \pi G_{N}\right)^{-1 / 2}$, where $G_{N}$ is Newton's gravitational constant. The sums over $j$ run over the two squark mass eigenstates. Left-right squark mixing is not relevant for this channel (nor are they for the other two channels), as explained in Appendix C. The total partonic cross section is obtained by integrating the above from $-\left(s-m_{\widetilde{g}}^{2}\right)$ to 0 . We have verified that our above result agrees with the differential cross section obtained in the second reference in Ref. [9] for $e^{+} e^{-} \rightarrow \chi \widetilde{G}$ (modulo color factors), where $\chi$ represents a photino.

The differential cross section for the subprocess $q g \rightarrow \widetilde{q_{j}} \widetilde{G}$ is given by $(j=1,2$ represents the two squark mass eigenstates)

$$
\begin{aligned}
d \hat{\sigma} / d t= & \frac{1}{6 \times 8 \times 16 \pi s^{2}} \frac{4 g_{s}^{2}}{M^{2} m_{3 / 2}^{2}}\left[-\frac{2\left(s-m_{\widetilde{q}_{j}}^{2}\right)^{2} s u}{3 s^{2}}-\frac{4 t^{4} u / s}{3\left[t-m_{\widetilde{q}_{j}}^{2}\right]^{2}}-\frac{2 u^{2}\left[\left(u-m_{\widetilde{q}_{j}}^{2}\right)\left(s-m_{\widetilde{q}_{j}}^{2}\right)+t\left(m_{\tilde{g}}^{2}-m_{\widetilde{q}_{j}}^{2}\right)\right]}{3\left[u-m_{\tilde{g}}^{2}\right]^{2}}-\frac{4 s t^{2} u}{3 s^{2}}\right. \\
& \left.-\frac{4 t^{2} u\left(s-m_{\widetilde{q}_{j}}^{2}\right)}{3 s\left[t-m_{\widetilde{q}_{j}}^{2}\right]}+\frac{4 u^{2} s\left(s-m_{\widetilde{q}_{j}}^{2}\right)}{3 s\left[u-m_{\widetilde{g}}^{2}\right]}+\frac{4 s t u\left(s-m_{\widetilde{q}_{j}}^{2}\right)}{3 s^{2}}+\frac{4 t^{2} u^{2}}{3\left[t-m_{\widetilde{q}_{j}}^{2}\right]\left[u-m_{\widetilde{g}}^{2}\right]}+\frac{8 t^{3} u}{3 s\left[t-m_{\widetilde{q}_{j}}^{2}\right]}-\frac{4 s t u^{2}}{3 s\left[u-m_{\widetilde{g}}^{2}\right]}\right] .
\end{aligned}
$$

The above cross section is new and has not been obtained elsewhere in the literature. The total partonic cross section is obtained by integrating the above from $-\left(s-m_{\widetilde{q}_{j}}^{2}\right)$ to 0 . When obtaining the total hadronic cross section for this channel we sum over both squark masses and then multiply the result by a factor of 2 to include the subprocess $g \bar{q} \rightarrow \widetilde{q}_{j}^{*} \widetilde{G}$.

The differential cross section for the subprocess $g g \rightarrow \widetilde{g} \widetilde{G}$ is given by

$$
\begin{aligned}
d \hat{\sigma} / d t= & \frac{1}{4 \times 64 \times 16 \pi s^{2}} \frac{24 g_{s}^{2}}{M^{2} m_{3 / 2}^{2}}\left[\frac{8\left(s-m_{\tilde{g}}^{2}\right) s t u}{3 s^{2}}+\frac{4}{3}\left(\frac{t^{3}\left(u+m_{\tilde{g}}^{2}\right)}{\left[t-m_{\tilde{g}}^{2}\right]^{2}}+\left(m_{\tilde{g}}^{2} t^{2} / s\right) \frac{t\left(t-m_{\tilde{g}}^{2}\right)+u\left(u-m_{\tilde{g}}^{2}\right)}{\left[t-m_{\tilde{g}}^{2}\right]^{2}}\right)\right. \\
& +\frac{4}{3}\left(\frac{u^{3}\left(t+m_{\tilde{g}}^{2}\right)}{\left[u-m_{\tilde{g}}^{2}\right]^{2}}+\left(m_{\tilde{g}}^{2} u^{2} / s\right) \frac{t\left(t-m_{\tilde{g}}^{2}\right)+u\left(u-m_{\tilde{g}}^{2}\right)}{\left[u-m_{\tilde{g}}^{2}\right]^{2}}\right)+\frac{8\left(s-m_{\tilde{g}}^{2}\right) s t u}{3 s^{2}}-\frac{8\left(s-m_{\tilde{g}}^{2} t^{2} u\right.}{3 s\left[t-m_{\tilde{g}}^{2}\right]}-\frac{8\left(s-m_{\tilde{g}}^{2}\right) t u^{2}}{3 s\left[u-m_{\tilde{g}}^{2}\right]} \\
& \left.+\frac{8\left(s-m_{\tilde{g}}^{2}\right) t^{2} u^{2} / s}{3\left[t-m_{\tilde{g}}^{2}\right]\left[u-m_{\tilde{g}}^{2}\right]}-\frac{8\left(s-m_{\tilde{g}}^{2}\right) t^{2} u}{3 s\left[t-m_{\tilde{g}}^{2}\right]}-\frac{8\left(s-m_{\tilde{g}}^{2}\right) t u^{2}}{3 s\left[u-m_{\tilde{g}}^{2}\right]}\right] .
\end{aligned}
$$

The total partonic cross section is obtained by integrating the above from $-\left(s-m_{\tilde{g}}^{2}\right)$ to 0 . The above differential cross section disagrees with the result in Ref. [10] by a factor of $\sqrt{2}$. However, if one replaces the definition of $\kappa$ $=\left(4 \pi G_{N}\right)^{1 / 2}$ in Ref. [10] by the "standard" definition $\kappa$ $=\left(8 \pi G_{N}\right)^{1 / 2}$ then our results agree.

While obtaining the above differential cross sections one must be careful while summing over polarization states of the incoming gluon(s). We have used

$$
\begin{aligned}
\sum_{\lambda=1,2} \epsilon^{\mu}\left(p_{1}, \lambda\right) \epsilon^{\nu}\left(p_{1}, \lambda\right) & =\sum_{\lambda=1,2} \epsilon^{\mu}\left(p_{2}, \lambda\right) \epsilon^{\nu}\left(p_{2}, \lambda\right) \\
& =-g^{\mu \nu}+\frac{2}{s}\left(p_{1}^{\mu} p_{2}^{\nu}+p_{1}^{\nu} p_{2}^{\mu}\right)
\end{aligned}
$$

where $p_{1,2}$ are the momenta of the incoming particles [16]. 
(For example, for $q g \rightarrow \widetilde{q} \widetilde{G}$, we pick the incoming quark momentum as $p_{2}$.) For $g g \rightarrow \widetilde{g} \widetilde{G}$ one could have alternatively used

$$
\sum_{\lambda=1,2} \epsilon^{\mu}\left(p_{1}, \lambda\right) \epsilon^{\nu}\left(p_{1}, \lambda\right)=\sum_{\lambda=1,2} \epsilon^{\mu}\left(p_{2}, \lambda\right) \epsilon^{\nu}\left(p_{2}, \lambda\right)=-g^{\mu \nu}
$$

and included the contribution of ghosts as in Ref. [16].

The hadronic cross section corresponding to any parton subprocess is given by

$$
\begin{aligned}
\sigma(S) & =\sum_{i, j} \int d x_{1} d x_{2} f_{i}\left(x_{1}, Q\right) f_{j}\left(x_{2}, Q\right) \hat{\sigma}_{i, j}\left[s, \alpha_{s}(\mu)\right] \\
& =\sum_{i, j} \int_{\tau_{0}}^{1} d \tau \int_{\tau}^{1} d x_{1}\left(1 / x_{1}\right) f_{i}\left(x_{1}, Q\right) f_{j}\left(\tau / x_{1}, Q\right) \hat{\sigma}_{i, j}
\end{aligned}
$$

Above $i, j$ run over all partons, valence and sea, that participate in the subprocess. $x_{i}=p_{i} / P_{i}$ is the ratio of the parton momentum to the hadron momentum. $\sqrt{S}$, the center of mass energy of the Tevatron, is $1.8 \mathrm{TeV} . \tau=x_{1} x_{2}, \tau_{0}=2 m_{\tilde{g}}^{2} / S$ for the $q \bar{q}$ and $g g$ channels, and $\tau_{0}=2 m_{\tilde{g}_{j}}^{2} / S$ for the $q g$ channel. For the $q \bar{q}$ and $g g$ channels we set the factorization scale $Q$ in the parton distribution functions $f_{i}$ to be the gluino mass, while for the $q g$ channel we set $Q$ to be the squark mass. We set the renormalisation scale $\mu$ equal to $m_{Z}$ and use the world average $\alpha_{s}\left(m_{Z}\right)=0.118$ in the modified minimal subtraction scheme.

\section{EXPERIMENTAL PREDICTIONS}

The three parton-level cross sections given in the previous section have been integrated over the parton distribution functions as indicated above. We assume both squark masses are equal for simplicity. We start with the $g g$ initiated process: $g g \rightarrow \widetilde{g} \widetilde{G}$, shown by the dashed lines in Fig. 4 , as a function of $m_{\tilde{g}}$, for a few choices of the gravitino mass (the cross section scales with $m_{\widetilde{G}}^{-2}$ ). For reference we also show the gluino pair-production cross section obtained in Ref. [10] (solid lines), which gives the order of magnitude of the traditional supersymmetric signals at hadron colliders. For low gluino masses the latter process dominates while for larger gluino masses the single gluino cross section dominates. Here we disagree with Ref. [10] that the $p \bar{p} \rightarrow \widetilde{g} \widetilde{g}$ cross section is greater than the cross section for $p \bar{p} \rightarrow \widetilde{g} \widetilde{G}$ via gluon fusion for $m_{\widetilde{G}} \gtrsim 10^{-5} \mathrm{eV}$.

The gluino-gravitino channel may also proceed from a quark-antiquark initial state $(q \bar{q} \rightarrow \widetilde{g} \widetilde{G})$. The hadronic cross section in this case depends on the squark mass in addition to the gluino mass. These are shown in Fig. 5 for $m_{\widetilde{G}}$ $=10^{-5} \mathrm{eV}$ and for various choices of the squark mass (solid lines), and also for the special case of $m_{\tilde{q}}=m_{\tilde{g}}$ (dotted line). In this figure we also show (dashed line) the additional contribution to this channel discussed above (i.e., from $g g \rightarrow \widetilde{g} \widetilde{G})$. It is evident that the $q \bar{q} \rightarrow \widetilde{g} \widetilde{G}$ channel generally dominates over the $g g \rightarrow \widetilde{g} \widetilde{G}$ channel for $m_{\widetilde{g}} \geq 200 \mathrm{GeV}$. This is at variance with the observation in Ref. [10] that

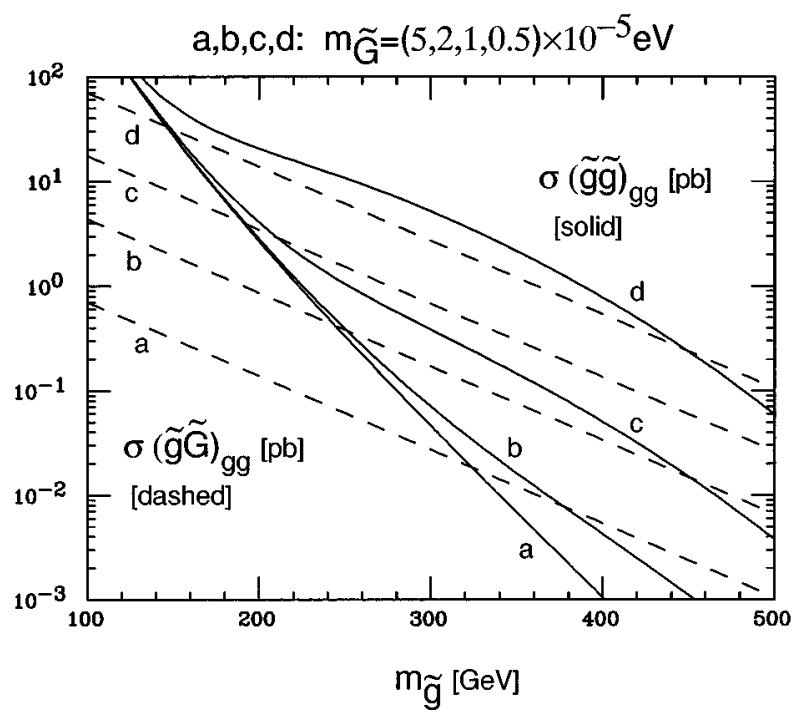

FIG. 4. Hadronic cross sections at the Tevatron that arise from the parton-level processes $g g \rightarrow \widetilde{g g}$ (solid lines) and $g g \rightarrow \widetilde{g} \widetilde{G}$ (dashed lines), as a function of $m_{\tilde{g}}$ for the indicated choices of the gravitino mass.

gluon fusion is the dominant subprocess. As noted in Ref. [10], the $p \bar{p} \rightarrow \widetilde{g} \widetilde{g}$ cross section via gluon fusion is fairly independent of the gravitino mass for $m_{\widetilde{G}}>10^{-5} \mathrm{eV}$ (see Fig. 4). Therefore, as the $p \bar{p} \rightarrow \widetilde{g} \widetilde{G}$ cross section scales with $m_{\widetilde{G}}^{-2}$, we may conclude from Fig. 5 that the latter process dominates over the former for gravitino masses as high as $m_{\widetilde{G}} \approx 0.3-1.0 \times 10^{-4} \mathrm{eV}$, depending on the gluino (and squark) mass. [Note that the results of Ref. [10] that we use for $\sigma(p \bar{p} \rightarrow \widetilde{g} \widetilde{g})$ include only the gluon fusion subprocess.]

The last channel to consider is that which originates from

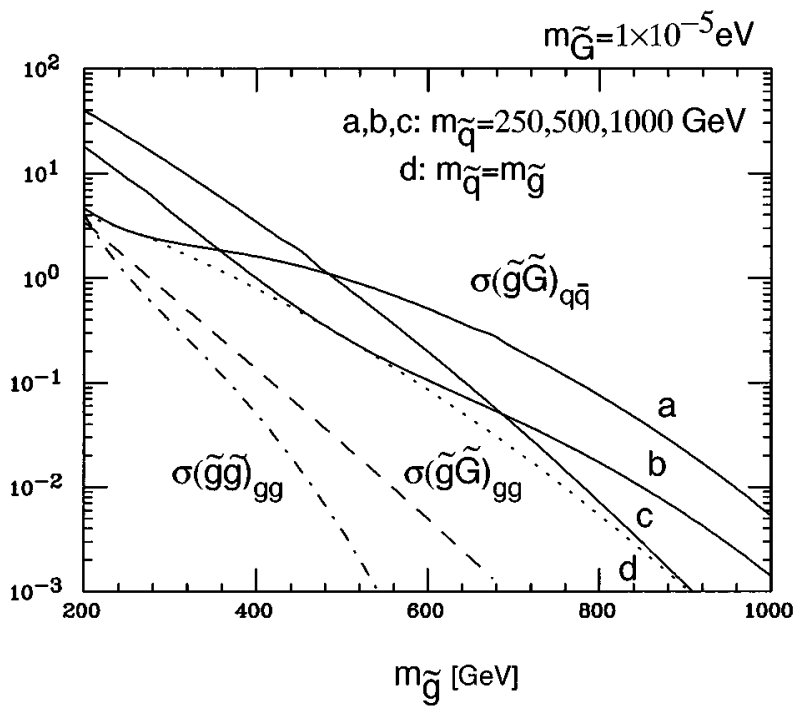

FIG. 5. Hadronic cross sections at the Tevatron that arise from the parton-level process $q \bar{q} \rightarrow \widetilde{g} \widetilde{G}$ (solid lines), as a function of $m_{\tilde{g}}$ for the indicated values of the squark mass, and of the gravitino mass (cross section scales as $m_{\tilde{G}}^{-2}$ ). Also shown for comparison are the corresponding cross sections via the $g g \rightarrow \widetilde{g} \widetilde{G}$ (dashed line) and $g g \rightarrow \widetilde{g} \widetilde{g}$ (dot-dashed line) subprocesses. 


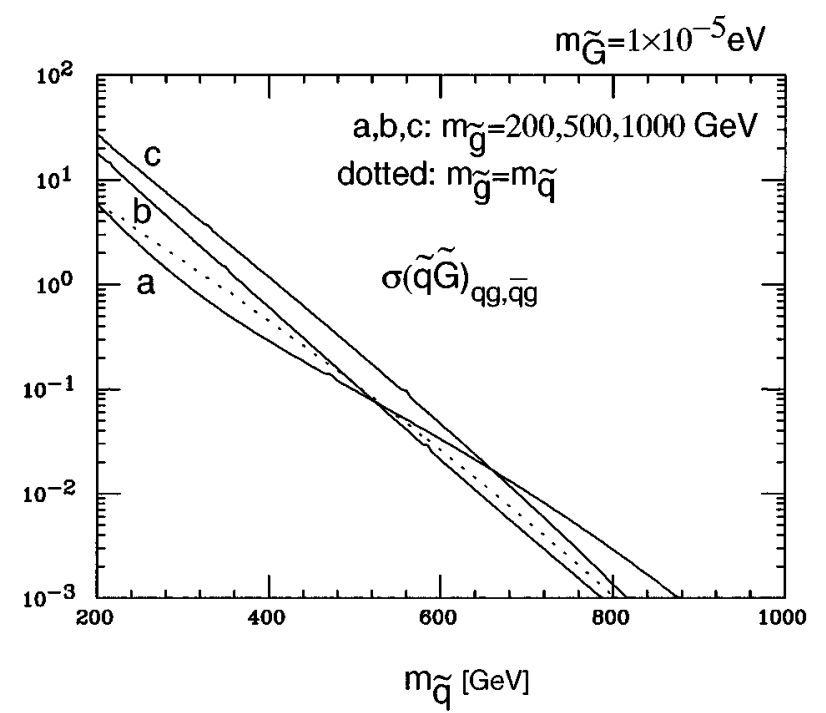

FIG. 6. Hadronic cross sections at the Tevatron that arise from the parton-level processes $q g, \bar{q} g \rightarrow \widetilde{q} \widetilde{G}, \widetilde{q}^{*} \widetilde{G}$, as a function of $m_{\widetilde{q}}$ for the indicated values of the gluino mass and of the gravitino mass (cross section scales as $m_{\tilde{G}}^{-2}$ ).

the parton-level processes $q g \rightarrow \widetilde{q} \widetilde{G}, \bar{q} g \rightarrow \widetilde{q}^{*} \widetilde{G}$. The corresponding cross section is shown in Figs. 6 and 7. For squark masses greater than $500 \mathrm{GeV}$ and gluino masses less than $500 \mathrm{GeV}$ the $q \bar{q}$ channel dominates over the $q g$ channel. However for low squark masses the $q g$ channel dominates.

Putting all light-gravitino signals together, one sees that these cross sections are higher than the traditional gluino pair-production one for gravitino masses as high as $m_{\widetilde{G}}$ $\approx 0.3-1.0 \times 10^{-4} \mathrm{eV}$, depending on the gluino (and squark) mass. For $m_{\widetilde{G}} \approx 10^{-5} \mathrm{eV}$, these cross sections have a kinematical reach in gluino/squark mass about twice as deep as the traditional gluino pair-production one. This reach decreases quickly with increasing gravitino mass, being surpassed by the traditional process for $m_{\tilde{G}} \approx 10^{-4} \mathrm{eV}$ and higher.

The study of standard model backgrounds to the above

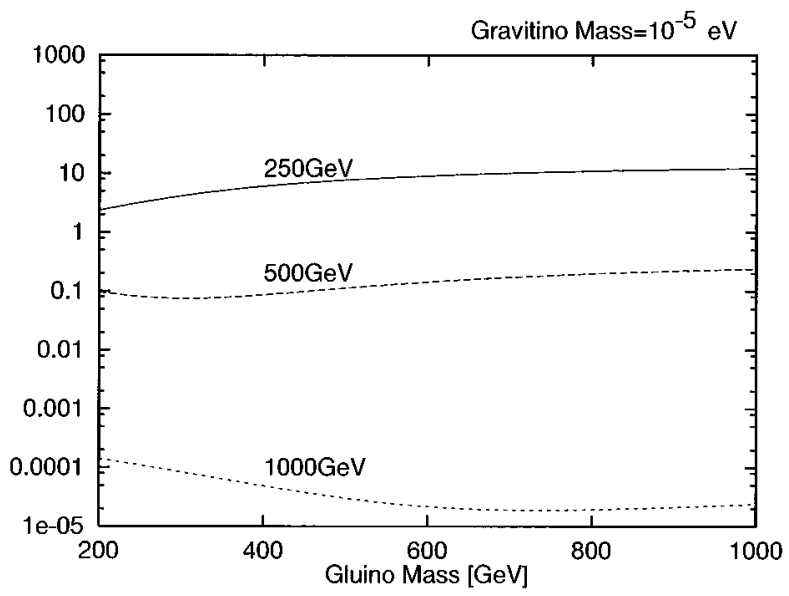

FIG. 7. Hadronic cross sections at the Tevatron that arise from the parton-level processes $q g, \bar{q} g \rightarrow \widetilde{q} \widetilde{G}, \widetilde{q}^{*} \widetilde{G}$, as a function of $m_{\widetilde{g}}$ for the indicated values of the squark mass and of the gravitino mass (cross section scales as $m_{\tilde{G}}^{-2}$ ). processes is beyond the scope of this paper, and in fact has been considered previously in the literature $[3,10]$. These authors have shown that on imposing suitable cuts, a sizeable signal may be observable over background. They then use limits on the multijet cross section at the Tevatron to exclude certain regions of the $\left(m_{\tilde{g}}, m_{\widetilde{G}}\right)$ parameter space. Here we simply comment that our signal calculations can exceed those in Ref. [10], and therefore one could expect an even more detectable signal than previously anticipated. The task of determining the actual observable signal is best left to the experimentalists. We hope that our calculations of the total cross sections will help in deciding whether these signals are worth pursuing in earnest.

\section{ACKNOWLEDGMENTS}

The work of J.K. and R.R. has been supported by the World Laboratory. The work of J.L. has been supported in part by U.S. DOE Grant No. DE-FG05-93-ER-40717 and that of D.V.N. by U.S. DOE Grant No. DE-FG05-91-ER40633. J.K. and R.R. would like to thank Takeo Moroi for many useful conversations. J.K. would like to thank J. Bagger for useful clarifications with respect to Ref. [17].

\section{APPENDIX A: COVARIANT DERIVATIVES}

We find it necessary to include the exact recipe for obtaining the Feynman rules from the relevant terms in the supergravity Lagrangian, since there are many conventions available in standard references. Problems arise when one uses Feynman rules from different sources without properly adapting them to a single scheme. For example, the conventions of Refs. [17-19] all differ from each other. For the future convenience of the reader, as well as ours, we provide a self-consistent set of Feynman rules and present the detailed steps involved in obtaining them, along with necessary comparisons with other references. Our goal is to provide a set of Feynman rules for interactions involving gravitinos that is consistent with Ref. [18].

We define our covariant derivative as

$$
\mathcal{D}_{\mu}=\partial_{\mu}+i g_{s} T^{A} A_{\mu}^{A},
$$

with commutation relation of $\mathrm{SU}(3)$ generators defined as

$$
\left[T^{A}, T^{B}\right]=i f^{A B C} T^{C},
$$

which lead to the tensor field

$$
F_{\mu \nu}^{A}=\partial_{\mu} A_{\nu}^{A}-\partial_{\nu} A_{\mu}^{A}-g_{s} f^{A B C} A_{\mu}^{B} A_{\nu}^{C} .
$$

These definitions follow those in Ref. [20], and differ from those in other texts. For example, in Ref. [21] the authors elect to use anti-Hermitian $T$ matrices, while Refs. [22] and [23] use Hermitian $T$ 's which, however, are negative of what we use. These different conventions result in different Feynman rules. For example, the Feynman rules for the quarkquark-gluon vertex are

$$
\begin{gathered}
-i g_{s} T^{A} A_{\mu}^{A}, \\
i g_{s} T^{A} A_{\mu}^{A},
\end{gathered}
$$




$$
g_{s} T^{A} A_{\mu}^{A},
$$

in Refs. [20], [22, 23], and [21], respectively. Similarly, the Feynman rule for the three-gluon vertex depends on the conventions used.

\section{APPENDIX B: CONVENTIONS}

Throughout the article, we use the flat space metric of

$$
\eta^{\mu \nu}=\operatorname{diag}(+1,-1,-1,-1) .
$$

We may form a numerically invariant tensor $\sigma^{\mu}$ :

$$
\left(\sigma^{\mu}\right)_{\alpha \dot{\beta}}=\left(I, \sigma^{i}\right), \quad i=1 \cdots 3,
$$

that transforms as a vector in $\mathrm{O}(1,3)$ using Pauli matrices $\sigma^{i}$,

$$
\sigma^{1}=\left(\begin{array}{cc}
0 & 1 \\
1 & 0
\end{array}\right) ; \quad \sigma^{2}=\left(\begin{array}{cc}
0 & -i \\
i & 0
\end{array}\right) ; \quad \sigma^{3}=\left(\begin{array}{cc}
1 & 0 \\
0 & -1
\end{array}\right)
$$

These are the Clebsch-Gordan coefficients which relate the $\left(\frac{1}{2}, \frac{1}{2}\right)$ of $\operatorname{SL}(2, C)$ to the vector of $\mathrm{O}(1,3)$. Here dotted indices transform under the $\left(0, \frac{1}{2}\right)$ of the Lorentz group, while those with undotted indices transform under the $\left(\frac{1}{2}, 0\right)$ conjugate representation. Spinors with upper and lower indices are related through the $\varepsilon$ tensor:

$$
\psi^{\alpha}=\varepsilon^{\alpha \beta} \psi_{\beta}, \quad \psi_{\alpha}=\varepsilon_{\alpha \beta} \psi^{\beta},
$$

where the antisymmetric tensor $\varepsilon$ 's are normalized as

$$
\begin{gathered}
\varepsilon^{\alpha \beta}=\left(\begin{array}{cc}
0 & 1 \\
-1 & 0
\end{array}\right), \\
\varepsilon_{\alpha \beta}=\left(\begin{array}{cc}
0 & -1 \\
1 & 0
\end{array}\right)=-\varepsilon^{\alpha \beta},
\end{gathered}
$$

which holds true for $\varepsilon$-tensors with dotted indices. The advantage of this scheme is that the mixed tensors are symmetric:

$$
\varepsilon_{\alpha \beta} \varepsilon^{\beta \gamma}=\delta_{\alpha}^{\gamma}
$$

These tensors are used to raise and lower the indices of the $\sigma$ matrices:

$$
\left(\bar{\sigma}^{\mu}\right)^{\dot{\alpha} \alpha}=\varepsilon^{\dot{\alpha} \dot{\beta}} \varepsilon^{\alpha \beta}\left(\sigma^{\mu}\right)_{\beta \dot{\beta}} .
$$

It is then straightforward to relate two-component spinors to four-component spinors through the realization of the Dirac $\gamma$ matrices:

$$
\gamma^{\mu}=\left(\begin{array}{cc}
0 & \sigma^{\mu} \\
\bar{\sigma}^{\mu} & 0
\end{array}\right)
$$

where

$$
\left(\bar{\sigma}^{\mu}\right)^{\dot{\alpha} \beta}=\left(I,-\sigma^{i}\right) .
$$

The generators of the Lorentz group in the spinor representation are given by

$$
\left(\sigma^{\mu \nu}\right)_{\alpha}^{\beta}=\frac{1}{4}\left(\sigma_{\alpha \dot{\alpha}}^{\mu} \bar{\sigma}^{\nu \dot{\alpha} \beta}-\sigma_{\alpha \dot{\alpha}}^{\nu} \bar{\sigma}^{\mu \dot{\alpha} \beta}\right)
$$

$$
\left(\bar{\sigma}^{\mu \nu}\right)_{\dot{\beta}}^{\dot{\alpha}}=\frac{1}{4}\left(\bar{\sigma}^{\mu \dot{\alpha} \alpha} \sigma_{\alpha \dot{\beta}}^{\nu}-\bar{\sigma}^{\nu \dot{\alpha} \alpha} \sigma_{\alpha \dot{\beta}}^{\mu}\right)
$$

With this choice of $\gamma$ matrices, Dirac spinors contain two Weyl spinors,

$$
\Psi_{D}=\left(\begin{array}{c}
\chi_{\alpha} \\
\bar{\psi}^{\alpha}
\end{array}\right)
$$

while Majorana spinors contain only one:

$$
\Psi_{M}=\left(\begin{array}{c}
\chi_{\alpha} \\
\bar{\chi}^{\alpha}
\end{array}\right)
$$

We define $\gamma^{5}$ as

$$
\gamma^{5}=i \gamma^{0} \gamma^{1} \gamma^{2} \gamma^{3}=\left(\begin{array}{cc}
-I & 0 \\
0 & I
\end{array}\right)
$$

Projection operators are defined accordingly:

$$
\begin{aligned}
& P_{L}=\frac{1}{2}\left(1-\gamma^{5}\right), \\
& P_{R}=\frac{1}{2}\left(1+\gamma^{5}\right) .
\end{aligned}
$$

\section{APPENDIX C: LAGRANGIAN AND FEYNMAN RULES}

We start with the general supergravity Lagrangian given in Chap. XXV of Ref. [17] and adapt it to the conventions listed in Appendix B. (Note that Ref. [17] uses the flat space metric diag $(-1,1,1,1)$, amongst other differences.) Below we explicitly write down the terms of the Lagrangian relevant to our calculation:

$$
\begin{aligned}
\mathcal{L}= & -g_{i j *} \widetilde{\mathcal{D}}_{\mu} A^{i} \widetilde{\mathcal{D}}^{\mu} A^{* j}-\frac{i}{2}\left(\bar{\lambda}^{A} \bar{\sigma}^{\mu} \widetilde{\mathcal{D}}_{\mu} \lambda^{A}+\lambda^{A} \sigma^{\mu} \widetilde{\mathcal{D}}_{\mu} \bar{\lambda}^{A}\right) \\
& -i g_{i j *} \bar{\chi}^{j} \bar{\sigma}^{\mu} \widetilde{\mathcal{D}}_{\mu} \chi^{i}+\sqrt{2} g_{s} g_{i j *}\left(X^{* j A} \chi^{i} \lambda^{A}+X^{i A} \bar{\chi}^{j} \bar{\lambda}^{A}\right) \\
& -\frac{1}{\sqrt{2} M} g_{i j *}\left[\widetilde{\mathcal{D}}_{\nu} A^{* j} \chi^{i} \sigma^{\mu} \bar{\sigma}^{\nu} \psi_{\mu}+\widetilde{\mathcal{D}}_{\nu} A^{i} \bar{\chi}^{j} \bar{\sigma}^{\mu} \sigma^{\nu} \bar{\psi}_{\mu}\right] \\
& -\frac{i}{2 M}\left[\psi_{\mu} \sigma^{a b} \sigma^{\mu} \bar{\lambda}^{A}+\bar{\psi}_{\mu} \bar{\sigma}^{a b} \bar{\sigma}^{\mu} \lambda^{A}\right] F_{a b}^{A},
\end{aligned}
$$




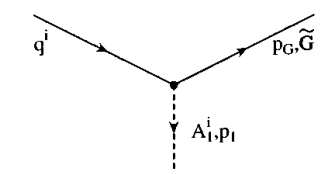

$2 /\left(3^{1 / 2} \mathrm{M} \mathrm{m}_{3 / 2}\right) \mathrm{p}_{1} \cdot \mathrm{p}_{\mathrm{G}}\left(\cos \theta \mathrm{P}_{\mathrm{L}}+\sin \theta \mathrm{P}_{\mathrm{R}}\right)$

(a)

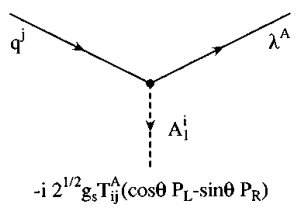

(c)

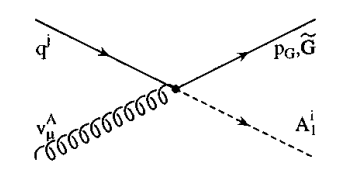

$-2 \mathrm{~g}_{s} /\left(3^{1 / 2} \mathrm{M} \mathrm{m}_{3 / 2}\right) \mathrm{T}_{\mathrm{ij}}^{\mathrm{A}} \mathrm{p}_{\mathrm{G}}^{\mu}\left(\cos \theta \mathrm{P}_{\mathrm{L}}+\sin \theta \mathrm{P}_{\mathrm{R}}\right)$

(e)

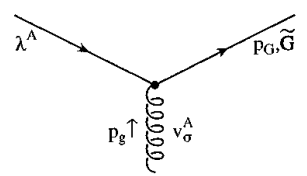

$1 /\left(26^{1 / 2} \mathrm{M} \mathrm{m}_{3 / 2}\right) \mathrm{p}_{g}^{\alpha} \mathrm{p}_{G}^{\beta}\left[\gamma_{\alpha}, \gamma_{\alpha}\right] \gamma_{\beta} \gamma^{5}$

(g)

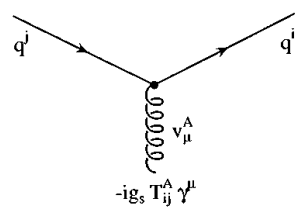

(i)

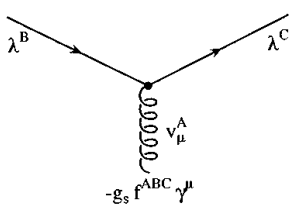

(k)

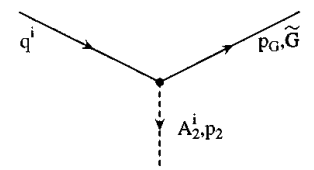

$2 /\left(3^{1 / 2} \mathrm{M} \mathrm{m}_{3 / 2}\right) \mathrm{p}_{2} \cdot \mathrm{p}_{\mathrm{G}}\left(\cos \theta \mathbf{P}_{\mathrm{R}}-\sin \theta \mathbf{P}_{\mathrm{L}}\right)$

(b)

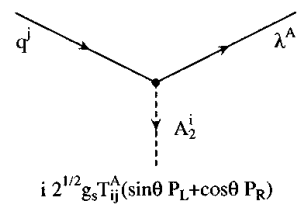

(d)

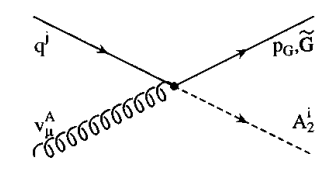

$-2 \mathrm{~g}_{\mathrm{s}} /\left(3^{1 / 2} \mathrm{M} \mathrm{m}_{3 / 2}\right) \mathrm{T}_{\mathrm{ij}}^{\mathrm{A}} \mathrm{p}_{\mathrm{G}}^{\mu}\left(\cos \theta \mathrm{P}_{\mathrm{R}}-\sin \theta \mathrm{P}_{\mathrm{L}}\right)$

(f)

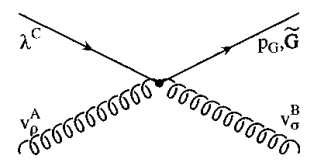

$\mathrm{i} /\left(26^{1 / 2} \mathrm{M} \mathrm{m}_{3 / 2}\right) \mathrm{g}_{\mathrm{s}} \mathrm{f}^{\mathrm{ABC}} \mathrm{PG}_{\mathrm{B}}^{\mathrm{B}}\left[\gamma_{\rho}, \gamma_{\sigma}\right] \gamma_{\beta} \gamma^{5}$

(h)

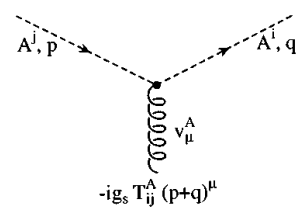

(j)

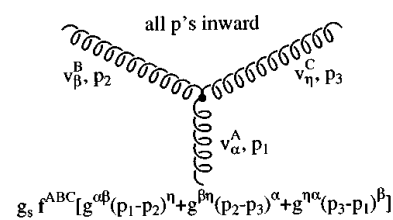

(I)

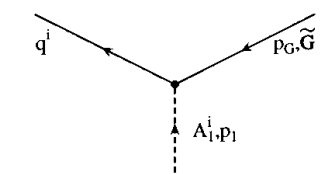

$-2 /\left(3^{1 / 2} \mathrm{M} \mathrm{m}_{3 / 2}\right) \mathrm{p}_{\mathrm{u}} \cdot \mathrm{P}_{\mathrm{G}}\left(\cos \theta \mathrm{P}_{\mathrm{R}}+\sin \theta \mathrm{P}_{\mathrm{L}}\right)$

(a')

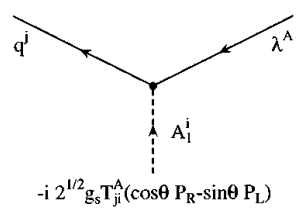

(c')

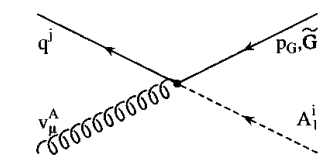

$2 \mathrm{~g}_{\mathrm{J}} /\left(3^{1 / 2} \mathrm{M} \mathrm{m}_{3 / 2}\right) \mathrm{T}_{\mathrm{jil}}^{A} \mathrm{PG}_{\mathrm{G}}^{\mu}\left(\cos \theta \mathrm{P}_{\mathrm{R}}+\sin \theta \mathrm{P}_{\mathrm{L}}\right.$

(e')

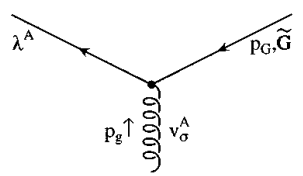

$1 /\left(26^{1 / 2} \mathrm{M} \mathrm{m}_{3 / 2}\right) \mathrm{pg}_{\mathrm{g}}^{\alpha} \mathrm{p}_{\sigma}^{\beta} \gamma_{\beta}^{5}\left[\gamma_{\sigma}, \gamma_{\alpha}\right]$

$\left(\mathrm{g}^{\prime}\right)$

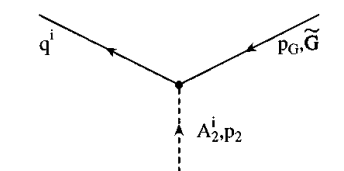

$-2 /\left(3^{1 / 2} \mathrm{M} \mathrm{m}_{3 / 2}\right) \mathrm{p}_{2} \cdot \mathrm{p}_{\mathrm{G}}\left(\cos \theta \mathrm{P}_{\mathrm{L}}-\sin \theta \mathrm{P}_{\mathrm{R}}\right)$

$\left(b^{\prime}\right)$

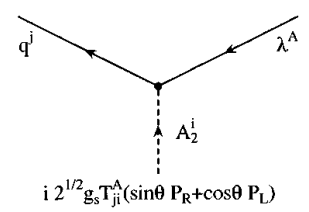

$\left(d^{\prime}\right)$

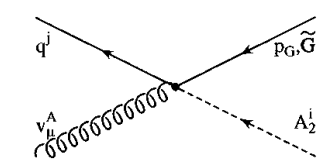

$2 \mathrm{~g}_{s} /\left(3^{1 / 2} \mathrm{M} \mathrm{m}_{3 / 2}\right) \mathrm{T}_{\mathrm{j}}^{\mathrm{A}} \mathrm{P}_{\mathrm{G}}^{\mu}\left(\cos \theta \mathrm{P}_{\mathrm{L}}-\sin \theta \mathrm{P}_{\mathrm{R}}\right)$

$\left(\mathbf{f}^{\prime}\right)$

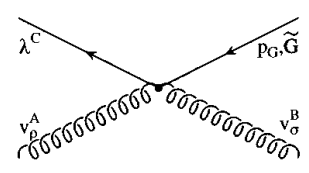

$-\mathrm{i} /\left(26^{1 / 2} \mathrm{M} \mathrm{m}_{3 / 2}\right) \mathrm{g}_{s} \mathrm{f}^{\mathrm{ABC}} \mathrm{p}_{G}^{\beta} \gamma^{5} \gamma_{\beta}\left[\gamma_{\sigma}, \gamma_{\rho}\right]$

$\left(h^{\prime}\right)$

FIG. 8. Feynman rules relevant to the processes discussed in the text. Arrows on scalars indicate direction of momentum, where relevant, and flow of particle flavor. Arrows on Majorana fermions also indicate direction of momentum, where relevant. Rules for primed diagrams are for Hermitian conjugates. Alternate rules for (a), (b), (e), (f), (g), and (h) using the gravitino couplings of Ref. [19] are provided in Table I.

where $g_{i j *}$ is the Kähler metric. $A^{i}$, s are scalar superpartners of chiral fermion $\chi^{i}$ s, $F^{A}$ are the usual field strength tensors of the gauge fields $v_{\mu}^{A}$ whose superpartners are gauginos $\lambda^{A}$, and $\psi_{\mu}$ is the gravitino field. Covariant derivative $\widetilde{\mathcal{D}}_{\mu}$ 's are

$$
\widetilde{\mathcal{D}_{\mu}} A^{i}=\partial_{\mu} A^{i}-g_{s} v_{\mu}^{A} X^{i A}
$$

$$
\begin{aligned}
& \widetilde{\mathcal{D}}_{\mu} \chi^{i}=\partial_{\mu} \chi^{i}-g_{s} v_{\mu}^{A} \frac{\partial X^{i A}}{\partial A^{j}} \chi^{j}, \\
& \widetilde{\mathcal{D}}_{\mu} \lambda^{A}=\partial_{\mu} \lambda^{A}-g_{s} f^{A B C} v_{\mu}^{B} \lambda^{C},
\end{aligned}
$$

where the Killing vectors $X^{i A}$ are defined in terms of the 
TABLE I. Alternate Feynman rules for gravitino couplings of Ref. [19] using our conventions. The index in parentheses refers to the diagram in Fig. 8. The rules for $(e),(f),\left(e^{\prime}\right)$, and $\left(f^{\prime}\right)$, which were not included in Ref. [19], are unchanged from Fig. 8.

\begin{tabular}{|c|c|}
\hline Vertex & Feynman rule \\
\hline Quark-squark -gravitino (a) $^{-}$ & $\frac{-\left(m_{A_{1}}^{2}-m_{q}^{2}\right)}{4 \sqrt{3} M m_{3 / 2}}\left(\cos \theta P_{L}+\sin \theta P_{R}\right)$ \\
\hline quark-squark ${ }_{2}$-gravitino (b) & $\frac{-\left(m_{A_{2}}^{2}-m_{q}^{2}\right)}{4 \sqrt{3} M m_{3 / 2}}\left(\cos \theta P_{R}-\sin \theta P_{L}\right)$ \\
\hline 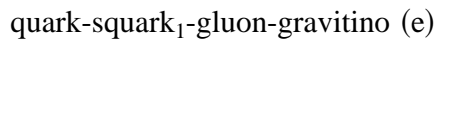 & $\frac{-2 g_{s}}{\sqrt{3} M m_{3 / 2}} T_{i j}^{A} p_{G}^{\mu}\left(\cos \theta P_{L}+\sin \theta P_{R}\right)$ \\
\hline quark-squark ${ }_{2}$-gluon-gravitino (f) & $\frac{-2 g_{s}}{\sqrt{3} M m_{3 / 2}} T_{i j}^{A} p_{G}^{\mu}\left(\cos \theta P_{R}-\sin \theta P_{L}\right)$ \\
\hline gluon-gluino-gravitino (g) & $\frac{m_{\lambda}}{2 \sqrt{6} M m_{3 / 2}} p_{g}^{\alpha}\left[\gamma_{\alpha}, \gamma_{\beta}\right] \gamma^{5}$ \\
\hline gluon-gluon-gluino-gravitino (h) & $\frac{-i m_{\lambda}}{2 \sqrt{6} M m_{3 / 2}} g_{s} f^{A B C}\left[\gamma_{\rho}, \gamma_{\sigma}\right] \gamma^{5}$ \\
\hline 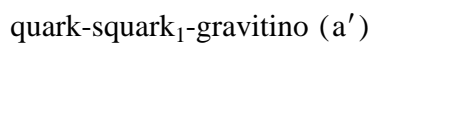 & $\frac{\left(m_{A_{1}}^{2}-m_{q}^{2}\right)}{4 \sqrt{3} M m_{3 / 2}}\left(\cos \theta P_{R}+\sin \theta P_{L}\right)$ \\
\hline quark-squark ${ }_{2}$-gravitino $\left(b^{\prime}\right)$ & $\frac{\left(m_{A_{2}}^{2}-m_{q}^{2}\right)}{4 \sqrt{3} M m_{3 / 2}}\left(\cos \theta P_{L}-\sin \theta P_{R}\right)$ \\
\hline 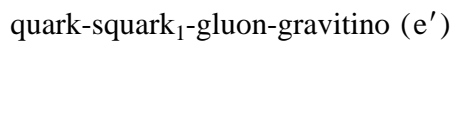 & $\frac{2 g_{s}}{\sqrt{3} M m_{3 / 2}} T_{j i}^{A} p_{G}^{\mu}\left(\cos \theta P_{R}+\sin \theta P_{L}\right)$ \\
\hline quark-squark ${ }_{2}$-gluon-gravitino $\left(\mathrm{f}^{\prime}\right)$ & $\frac{2 g_{s}}{\sqrt{3} M m_{3 / 2}} T_{j i}^{A} p_{G}^{\mu}\left(\cos \theta P_{L}-\sin \theta P_{R}\right)$ \\
\hline gluon-gluino-gravitino $\left(\mathrm{g}^{\prime}\right)$ & $\frac{m_{\lambda}}{2 \sqrt{6} M m_{3 / 2}} p_{g}^{\alpha} \gamma^{5}\left[\gamma_{\beta}, \gamma_{\alpha}\right]$ \\
\hline gluon-gluon-gluino-gravitino $\left(\mathrm{h}^{\prime}\right)$ & $\frac{i m_{\lambda}}{2 \sqrt{6} M m_{3 / 2}} g_{s} f^{A B C} \gamma^{5}\left[\gamma_{\sigma}, \gamma_{\rho}\right]$ \\
\hline
\end{tabular}

Killing potential $D^{A}$. For the minimal Kähler potential $K$ $=A^{i *} A^{i}$, the Killing vectors and the Killing potential take the form of

$$
\begin{gathered}
X^{i A}=-i g^{i j *} \frac{\partial}{\partial A^{j *}} D^{A}=-i T_{j i}^{A} A^{i}, \\
X^{j * A}=i g^{i j *} \frac{\partial}{\partial A^{i}} D^{A}=i A^{j *} T_{j i}^{A}, \\
D^{A}=A^{j *} T_{j i}^{A} A^{i} .
\end{gathered}
$$

Four-spinors are constructed using two-spinors as

$$
q^{(D)}=\left(\begin{array}{c}
\chi_{1 \alpha} \\
\bar{\chi}_{2}^{\dot{\alpha}}
\end{array}\right), \quad \bar{q}^{(D)}=\left(\chi_{2}^{\alpha}, \bar{\chi}_{1 \dot{\alpha}}\right)
$$

$$
\begin{gathered}
\lambda^{(M)}=\left(\begin{array}{c}
-i \lambda_{\alpha} \\
i \bar{\lambda}^{\dot{\alpha}}
\end{array}\right), \quad \bar{\lambda}^{(M)}=\left(-i \lambda^{\alpha}, i \bar{\lambda}_{\dot{\alpha}}\right), \\
\psi^{(M)}=\left(\begin{array}{c}
\psi_{\alpha} \\
\bar{\psi}^{\alpha}
\end{array}\right), \quad \bar{\psi}^{(M)}=\left(\psi^{\alpha}, \bar{\psi}_{\dot{\alpha}}\right),
\end{gathered}
$$

where superscript $(D)$ is for Dirac spinors, and $(M)$ for Majorana spinors. The extra $i$ 's in gauginos are introduced following Ref. [18]. After adding appropriate mass terms to Eq. (C1), we obtain the Lagrangian in four-spinor notation:

$$
\mathcal{L}=\mathcal{L}_{0}+\mathcal{L}_{1}+\mathcal{L}_{2}+\mathcal{L}_{3}+\mathcal{L}_{4}+\mathcal{L}_{5}+\mathcal{L}_{6}+\mathcal{L}_{7}+\mathcal{L}_{8}
$$

where $\mathcal{L}_{0}$ includes kinetic terms

$$
\mathcal{L}_{0}=\vec{q}^{i}\left(i \not \partial-m_{q}\right) q^{i}-A^{i}\left(\square+m_{A}^{2}\right) A^{i *}+\frac{1}{2}\left[\bar{\lambda}^{A}\left(i \not b-m_{\lambda}\right) \lambda^{A}\right],
$$


and others include three- and four-particle interaction terms

$$
\begin{gathered}
\mathcal{L}_{1}=-g_{s} T_{i j}^{A} \bar{q}^{i} \boldsymbol{v}^{A} q^{j}, \\
\mathcal{L}_{2}=-g_{s} v_{\mu}^{A} T_{i j}^{A}(p+q)^{\mu} A^{j} A^{i *}, \\
\mathcal{L}_{3}=-\frac{i}{2} g_{s} f^{A B C} \bar{\lambda}^{A} v^{B} \lambda^{C}, \\
\mathcal{L}_{4}=-\sqrt{2} g_{s} T_{j i}^{A}\left(A_{L}^{j *} \bar{\lambda}^{A} P_{L} q^{i}-A_{R}^{j *} \bar{\lambda}^{A} P_{R} q^{i}+\text { H.c. }\right), \\
+\frac{1}{\sqrt{2} M}\left(\partial_{\nu} A_{L}^{i *} \bar{\psi}_{\mu} \gamma^{\nu} \gamma^{\mu} P_{L} q^{i}+\partial_{\nu} A_{R}^{i *} \bar{\psi}_{\mu} \gamma^{\nu} \gamma^{\mu} P_{R} q^{i}\right. \\
\mathcal{L}_{6}=-\frac{\mathrm{C} 10),}{\sqrt{2} g_{s} v_{\nu}^{A} T_{j i}^{A}}\left(A_{L}^{j *} \bar{\psi}_{\mu} \gamma^{\nu} \gamma^{\mu} P_{L} q^{i}+A_{R}^{j *} \bar{\psi}_{\mu} \gamma^{\nu} \gamma^{\mu} P_{R} q^{i}\right. \\
+ \text { H.c. }), \\
\mathcal{L}_{7}=-\frac{i}{8 M}\left[\bar{\psi}_{\mu}\left[\gamma^{\alpha}, \gamma^{\beta}\right] \gamma^{\mu} \lambda^{A}\right] F_{\alpha \beta}^{A},
\end{gathered}
$$

from which our Feynman rules will be derived. Note that an extra factor of 2 is necessary in the Feynman rules to account for the Majorana nature of gauginos and gravitinos. Mixing between left- and right-squarks is proportional to the mass of their quark counterpart. In our calculation of cross sections at the Fermilab $p-\bar{p}$ collider, the effect of mixing is practically nonexistent. Therefore we replace $A_{L}$ and $A_{R}$ with the mass eigenstates $A_{1}$ and $A_{2}$. Relative signs between terms within Eqs. (C13) $-(\mathrm{C} 15)$ are related to the definition of the fermions in Eqs. (C5) and (C6), and to the fact that $A_{R}$ 's transform as antitriplets of $\mathrm{SU}(3)_{c}$, i.e., their generators are $-T^{A *}$ instead of $T^{A}$ (see pp. 208 and 223 of Ref. [18]). Terms (C13)-(C15) include interactions with gravitinos. If the gravitino is very light, which is the scenario we are pursuing, spin- $\frac{3}{2}$ components of the gravitino decouple from the spin- $\frac{1}{2}$ components, and it interacts with matter as a massless goldstino with derivative couplings. Thus we may use an effective Lagrangian with a massless Goldstino $\psi$ by making the substitution

$$
\psi_{\mu} \simeq i \sqrt{\frac{2}{3}} \frac{1}{m_{3 / 2}} \partial_{\mu} \psi
$$

After simple rearrangements, terms $(\mathrm{C} 13)-(\mathrm{C} 15)$ become

$$
\begin{gathered}
\frac{2 i}{\sqrt{3} M m_{3 / 2}}\left(p_{1} \cdot p_{G} A_{1}^{i *} \bar{\psi}_{G} P_{L} q^{i}+p_{2} \cdot p_{G} A_{2}^{i *} \bar{\psi}_{G} P_{R} q^{i}+\text { H.c. }\right) \\
-\frac{2 i g_{s} v^{A} \cdot p_{G} T_{j i}^{A}}{\sqrt{3} M m_{3 / 2}}\left(A_{1}^{i *} \bar{\psi}_{G} P_{L} q^{i}+A_{2}^{i *} \bar{\psi}_{G} P_{R} q^{i}+\text { H.c. }\right) \\
-\frac{i}{2 \sqrt{6} M m_{3 / 2}}\left(\bar{\psi}_{G}\left[p_{g}, \boldsymbol{v}^{A}\right] p_{G} \gamma^{5} \lambda^{A}\right) \\
-\frac{g_{s} f^{A B C}}{4 \sqrt{6} M m_{3 / 2}}\left(\bar{\psi}_{G}\left[\boldsymbol{\mho}^{B}, \boldsymbol{v}^{C}\right] p_{G} \gamma^{5} \lambda^{A}\right)
\end{gathered}
$$

where subscripts $1,2, G$, and $g$ of the momenta are for squark $_{1}$, squark ${ }_{2}$, gravitino, and gluino, respectively. Resulting Feynman rules are listed in Fig. 8. For the sake of completeness, we include the effects of left-right squark mixing in the Feynman rules, where $\theta=0$ implies no mixing.

In Sec. 4.5 of Ref. [19], the author uses the on-shell condition of external particles to replace derivatives in the Lagrangian by masses of external particles. This replacement is valid for internal particles as well as off-shell contributions cancel, which is characteristic of the effective Lagrangian. In Table I we provide the Feynman rules for gravitino couplings obtained by applying this prescription. Our rules appear different from those in Ref. [19] because of the different conventions that we use (note the differences in the definitions of $\gamma^{\mu}$ and $\gamma^{5}$, and gluinos). Note that the four-point interaction quark-squark-gluon-gravitino was overlooked in Ref. [19].
[1] P. Fayet, Phys. Lett. 69B, 489 (1977); 70B, 461 (1977); 84B, 421 (1979); 86B, 272 (1979); Phys. Lett. B 175, 471 (1986).

[2] J. Ellis, K. Enqvist, and D. V. Nanopoulos, Phys. Lett. 147B, 99 (1984).

[3] D. Dicus, S. Nandi, and J. Woodside, Phys. Rev. D 41, 2347 (1990); 43, 2951 (1991).

[4] D. Stump, M. Wiest, and C.-P. Yuan, Phys. Rev. D 54, 1936 (1996); S. Dimopoulos, M. Dine, S. Raby, and S. Thomas, Phys. Rev. Lett. 76, 3494 (1996); S. Dimopoulos, S. Thomas, and J. Wells, Phys. Rev. D 54, 3283 (1996); K. Babu, C. Kolda, and F. Wilczek, Phys. Rev. Lett. 77, 3070 (1996).

[5] S. Ambrosanio, G. Kane, G. Kribs, S. Martin, and S. Mrenna, Phys. Rev. Lett. 76, 3498 (1996); Phys. Rev. D 54, 5395 (1996).

[6] J. L. Lopez and D. V. Nanopoulos, Mod. Phys. Lett. A 11, 2473 (1996); Phys. Rev. D 55, 4450 (1997).
[7] S. Park, in Proceedings of the 10th Topical Workshop on Proton-Antiproton Collider Physics, Fermilab, 1995, edited by R. Raja and J. Yoh (AIP, New York, 1995), p. 62.

[8] D. Dicus, S. Nandi, and J. Woodside, Phys. Lett. B 258, 231 (1991).

[9] J. L. Lopez, D. V. Nanopoulos, and A. Zichichi, Phys. Rev. Lett. 77, 5168 (1996); Phys. Rev. D 55, 5813 (1997).

[10] D. Dicus and S. Nandi, Phys. Rev. D 56, 4166 (1997).

[11] J. Ellis, K. Enqvist, and D. V. Nanopoulos, Phys. Lett. 151B, 357 (1985); M. Nowakowski and S. D. Rindani, Phys. Lett. B 348, 115 (1995); T. Gherghetta, Nucl. Phys. B485, 25 (1997); J. Grifolds, R. Mohapatra, and A. Riotto, Phys. Lett. B 401, 283 (1997); 400, 124 (1997).

[12] A. Brignole, F. Feruglio, and F. Zwirner, hep-ph/9703286.

[13] M. A. Luty and E. Ponton, hep-ph/9706268.

[14] M. Drees and J. Woodside, in Proceedings of the ECFA Large 
Hadron Collider Workshop, Aachen, Germany, 1990, edited by G. Jarlskog and D. Rein (CERN Report No. 90-10, Geneva, Switzerland, 1990), p. 681.

[15] T. Bhattacharya and P. Roy, Phys. Lett. B 206, 655 (1988); Nucl. Phys. B328, 469 (1989); B328, 481 (1989); R. Casalbuoni, S. de Curtis, D. Dominici, F. Feruglio, and R. Gatto, Phys. Lett. B 216, 325 (1989).

[16] J. Babcock, D. Sivers, and S. Wolfram, Phys. Rev. D 18, 162 (1978), see Appendix B.

[17] J. Wess and J. Bagger, Supersymmetry and Supergravity, 2nd ed. (Princeton University Press, Princeton, 1992).
[18] H. Haber and G. Kane, Phys. Rep. 117, 75 (1985).

[19] T. Moroi, Ph.D. thesis, Tohoku University, Japan.

[20] I. J. R. Aitchison and A. J. G. Hey, Gauge Theories in Particle Physics, 2nd ed. (Adam Hilger, Philadelphia, 1989).

[21] C. Itzykson and J. Zuber, Quantum Field Theory (McGrawHill, New York, 1985).

[22] S. Pokorski, Gauge Field Theories (Cambridge University Press, Cambridge, England, 1987).

[23] T. Cheng and L. Li, Gauge Theory of Elementary Particle Physics (Oxford University Press, Oxford, 1984). 